\title{
1 Near-infrared chemical imaging and its correlation with \\ 2 the mechanical properties of chitosan-gelatin edible films
}

4 Palma, F. ${ }^{1}$, Michniak-Kohn, B. ${ }^{2}$, Pérez-Correa, J. R. ${ }^{3}$, Hernandez, E. ${ }^{4}$, Romañach, R. ${ }^{5}$,

5 and Valenzuela, L.M. ${ }^{6,7^{*}}$

6

$7 \quad{ }^{1}$ Department of Chemical and Bioprocesses Engineering, ASIS-UC Interdisciplinary

8 Research Program on Tasty, Safe and Healthy Foods, Pontificia Universidad Católica de

9 Chile, Avenida Vicuña Mackenna 4860, Santiago, Chile. fvpalma@uc.cl

$10{ }^{2}$ Ernest Mario School of Pharmacy, Rutgers, The State University of New Jersey,

11 Piscataway, NJ 08854, USA. michniak@dls.rutgers.edu

$12{ }^{3}$ Department of Chemical and Bioprocesses Engineering, ASIS-UC Interdisciplinary

13 Research Program on Tasty, Safe and Healthy Foods, Pontificia Universidad Católica de

14 Chile, Avenida Vicuña Mackenna 4860, Santiago, Chile. perez@ing.puc.cl

$15{ }^{4}$ Department of Chemistry, University of Puerto Rico, Mayagüez Campus, Mayagüez, PR

16 00681, USA. eduardo.hernandez1@upr.edu

$17{ }^{5}$ Department of Chemistry, University of Puerto Rico, Mayagüez Campus, Mayagüez, PR

18 00681, USA. rodolfoj.romanach@upr.edu

*Corresponding author: L. M. Valenzuela, PhD Vicuña Mackenna 4860, Santiago, Chile.

${ }^{7}$ Research Center for Nanotechnology and Advanced Materials “'CIEN-UC', Pontificia Universidad Católica de Chile, Santiago, Chile.

Tel.: +562-2354-1227

E-mail: $\underline{\text { lvalenzr@ing.puc.cl }}$ 


\section{ABSTRACT}

32 Plasticizers influence the physical properties of edible films by their interaction with the

33 film-forming polymers. Using near-infrared chemical imaging, it is possible to

34 characterize the interaction between compounds through the analysis of their relative

35 presence throughout the film (abundance) and their variability. These parameters and

36 standard mechanical properties were used to characterize the interaction between gelatin,

37 chitosan and several plasticizers, pure or in binary combinations. Triacetin showed the

38 least interaction with the polymers, while polyethylene glycol 400 and glycerol showed

39 high interaction with them. In addition, we observed that the tensile strength of the film

40 was well correlated with the variability of gelatin and chitosan.

41

\section{Keywords}

43 Hydrocolloid; near-infrared spectroscopy; plasticizer; molecular interaction; tensile

44 strength 


\section{1. INTRODUCTION}

63 Edible films are thin layers of a polymer material that control the solute exchange

64 between the matrix and the environment, thus extending the shelf life of a food (Pavlath

$65 \&$ Orts, 2009). To achieve this, the films' polymer matrix should have the necessary

66 physical-chemical properties for the specific food with which it will be used. Edible films

67 can be created to have the necessary mechanical and structural properties with the use of

68 hydrocolloid molecules, which are hydrophobic biopolymers with high molecular weight

69 and long polymeric chains (Falguera, Quintero, Jiménez, Muñoz, \& Ibarz, 2011). The

70 most used compound for the manufacturing of edible films is gelatin, mainly due to its

71 ability to form films at low temperatures (Gómez-Estaca, Gómez-Guillén, Fernández-

72 Martín, \& Montero, 2011), and chitosan, for being antibacterial, biodegradable,

73 biocompatible, non-toxic and renewable (van den Broek, Knoop, Kappen, \& Boeriu,

74 2015).

75

76 Edible films are made up of molecular interactions between the polymers used in the

77 film, including covalent bonds (i.e. disulfide interactions and cross-linking) as well as

78 ionic bonds, hydrogen bonds and electrostatic interactions. These interactions generate

79 films that are rigid and brittle, providing less protection to the food (Cao, Yang, \& Fu,

80 2009; Rivero, García, \& Pinotti, 2010). It is possible to improve the stability and

81 performance of the edible films with the addition of plasticizers (Talja, Helén, Roos, \&

82 Jouppila, 2008). Plasticizers are compounds that reduce inter-molecular forces and

83 increase the mobility of the polymeric chains of the film (Sothornvit \& Krochta, 2005).

84 The plasticizers interact with the polymers of the matrix through van der Waals forces,

85 hydrogen bonds and weak electrostatic interactions (Marcilla, García, \& Beltrán, 2012).

86 The amount of oxygen atoms per molecule, the molecular weight and polarity (dielectric

87 constant) are the characteristics of the plasticizer that determine the type and level of

88 interaction with the film-forming polymers (Immergut \& Mark, 1965). Also, the addition

89 of high concentrations of hydrophilic plasticizers (i.e., over $20 \%$ in chitosan films,

90 (Suyatma, Tighzert, \& Copinet, 2005)) could over-plasticize the films, producing phase

91 separation (Arvanitoyannis, Nakayamab, \& Aibab, 1998). Over-plasticized films are

92 sticky, weak and extend more than required. Sometimes exudate drops can be observed in 
93 the surface of over-plasticized films (Taylor, Taylor, Belton, \& Minnaar, 2009; Wilson,

94 1995).

95

96 Infrared spectroscopy (IR) is a good option to study the chemical interaction between

97 compounds (Lefevre \& Subirade, 2001). IR spectroscopy provides information about the

98 internal chemical structure of the sample, for instance, the type or quantity of a given

99 bond that is present in the sample (Zapf, 2009). This is achieved by detecting chemical

100 bonds through the rotation and vibration that they show in a given spectral range of the

101 electromagnetic spectrum (13000-10 $\mathrm{cm}^{-1}$ or $0.76-1000 \mu \mathrm{m}$, depending on the units

102 used) (Gendrin, Roggo, \& Collet, 2008). One type of IR is near-infrared spectroscopy

103 (NIR), used mainly to study the overtones and combinations of $\mathrm{CH}, \mathrm{OH}, \mathrm{NH}$ bands and

104 stretching, as well as bond vibration modes that provide highly specific molecular

105 information for species identification (Furukawa, Sato, Shinzawa, Noda, \& Ochiai,

106 2007).

107

108 Near-infrared chemical imaging (NIR-CI) techniques apply conventional near-infrared

109 spectroscopy, together with a system that captures multiple images through many

110 wavelengths. The resulting image is a tri-dimensional (3D) matrix or hyperspectral data

111 cube, with two spatial dimensions and one spectral dimension (Gendrin et al., 2008). The

112 conventional NIR technique only provides the average spectrum of the surface of each

113 sample; however, NIR-CI provides a spectrum of each pixel of the samples' entire

114 surface, allowing a complete chemical characterization with a quick and non-invasive

115 process.

116

117 With the information obtained by the NIR-CI, together with the use of chemometric

118 tools, a characterization can be created with the distribution of the samples' compound of

119 interest (Sacré et al., 2014), obtaining the histogram parameters of the compounds'

120 intensities: mean, standard deviation, kurtosis and skew. The mean of the distribution

121 values corresponds to the abundance of the compounds on the surface of the sample (de

122 Juan et al., 2004). The standard deviation of the distribution is defined as the variability

123 of the abundance of the samples' compounds (Puchert, Lochmann, Menezes, \& Reich, 
124 2010). Kurtosis measures the symmetrical tailing or 'peakedness' of the distribution,

125 where zero indicates a normal distribution. Finally, skew is the measurement of the

126 asymmetry of the distribution, with zero corresponding to a symmetric distribution (E. N.

127 Lewis, Schoppelrei, Lee, \& Kidder, 2007; Montgomery \& Runger, 2003). Viewed

128 together, the variability of abundance, the degree of unimodal distribution and the

129 asymmetry of the distribution are descriptors of the homogeneity of the sample.

130 Moreover, these parameters depend on the spectral information of the sample, which

131 changes in response to the interaction between the components. (Lefevre \& Subirade, 132 2001).

134 In pharmaceutics, there are studies that relate physical properties of drug matrices with 135 the distribution and abundance of ingredients for the NIR-CI analysis. For example,

136 Ellison, Ennis, Hamad, and Lyon (2008) showed that NIR-CI can be an effective tool for 137 monitoring physical quality, distribution of active pharmaceutical ingredient in the 138 tablets, and also their uniform compaction force. Y. F. Wang et al. (2014) reported 139 correlation models that compared tablet hardness and spatial distribution, obtained using

140 NIR-CI. Jérez Rozo et al. (2011) used NIR-CI to characterize the spatial distribution and

141 abundance of the film-forming polymer and the drug contained in the film used in a

142 pharmaceutical application. Both parameters were correlated with the size of the particle

143 and the agglomeration of the drug, allowing them to determine the dissolution speed and

144 drug uniformity obtained with the film. This suggests that the abundance and distribution

145 of the polymeric film compounds obtained by NIR-CI, may be related to the films'

146 physico-chemical properties.

148 Since the mechanical properties of edible films are determined by the interaction between

149 the plasticizers and the film-forming polymers (Bergo \& Sobral, 2007), these properties

150 could be analyzed with NIR-CI. This paper investigates the relationship between the

151 abundance of the compounds and their variability, as obtained with NIR-CI, and the

152 mechanical properties of the gelatin and chitosan edible films. Our objective is to

153 research the potential of NIR-CI to qualitatively and quantitatively characterize, in a non- 
154 invasive way, the homogeneity of the edible films and its impact on the films' 155 mechanical properties. 


\section{2. MATERIALS AND METHODS}

\section{$157 \quad 2.1$ Materials}

159 The film forming solutions were fabricated using gelatin from cold water fish skin

160 (Sigma-Aldrich Lot\# SLBG7701V, average molecular mass $60 \mathrm{kDa}$; bloom number: 225

$161-325$; viscosity at $24^{\circ} \mathrm{C}, 31500 \mathrm{cps}$ ), and chitosan from Pandalus borealis (Sigma-

162 Aldrich Lot\# MKBD4275V; medium molecular weight; 77\% deacetylation, viscosity 450

$163 \mathrm{mPas}$ at $1 \%$ concentration in $1 \%$ acetic acid). The plasticizers used were glycerol ( $\geq 99 \%$,

164 Sigma-Aldrich), sorbitol ( $\geq 99.5 \%$, Sigma-Aldrich), polyethylene glycol-400 (PEG400)

165 (EMD Millipore), and triacetin (99\%, Acros Organics).

166

167

2.2 Methods

168

169

2.2.1 Film forming solutions

170 The film- forming solutions were fabricated by dissolving chitosan in acetic acid at $1 \%$

$171 \mathrm{v} / \mathrm{v}$ to reach a concentration of $1.55 \% \mathrm{w} / \mathrm{w}$. The dissolved chitosan was mixed with liquid 172 state gelatin at $2.3 \% \mathrm{w} / \mathrm{w}$. In each case, a plasticizer was added at $1.16 \% \mathrm{w} / \mathrm{w}$ (Hosseini,

173 Rezaei, Zandi, \& Ghavi, 2013), or two plasticizers at $0.58 \%$ w/w each (Table 1). Gelatin

174 and chitosan film, without the addition of plasticizers, was used as control film. Each

175 film-forming solution was stirred until dissolved, and then was heated and maintained at

$17645^{\circ} \mathrm{C}$ for $1 \mathrm{~h}$, being constantly stirred. The resulting solution had a pH of $4.566 \pm 0.007$

177 and was filtered using vacuum and stored at room temperature until the edible films'

178 fabrication.

179

180 Table 1. Formulation of edible films used in this study

\begin{tabular}{|c|c|c|}
\hline Compound & (g/100 g sol.) & Percentagk81 \\
\hline Gelatin & 2.30 & 46182 \\
\hline Chitosan & 1.55 & $\begin{array}{ll}31 & 184\end{array}$ \\
\hline Plasticizers $^{(2)}$ & 1.16 & $23 \quad 185$ \\
\hline
\end{tabular}

$187{ }^{(1)}$ Percentage corresponds to composite grams/grams of total compounds in the film multiplied by 100.

188 (2) Plasticizers correspond to glycerol, sorbitol, PEG400, and triacetin, either as a single plasticizer or as a

189 combination of two of them in a proportion $1: 1$. 


\subsubsection{Fabrication of edible films}

192 Solvent casting was used to produce edible films. Aliquots of $5.4 \mathrm{~g}$ of a film-forming

193 solution were poured into petri dishes with surface areas of $24 \mathrm{~cm}^{2}$, and dried at $30^{\circ} \mathrm{C}$ and

$19425 \pm 5 \%$ relative humidity in a system of forced air until a stable mass was reached (15

195 h). The average films' thickness was $100 \pm 23 \mu \mathrm{m}$.

\subsubsection{Near-infrared chemical images (NIR-CI)}

198 A Malvern Instrument Spectral Dimension SyNIRgi spectrometer chemical imaging

199 system (Malvern, UK) was used. The Malvern system includes a focal plan array (FPA)

200 mode with a tunable liquid-crystal filter that allowed full field analysis.

202 The edible films were placed over a white ceramic disk with a $28 \mathrm{~mm}$ diameter, which

203 was used as a reference for the acquisition system. Spectra and images were obtained in

204 transflectance mode. In this mode, the radiation passes through the bulk of the film,

205 bounces back in the highly reflective ceramic, passes through the bulk of the film again

206 and then to the detector (Li Yoon et al., 1999). Therefore, the transflectance mode

207 evaluates not only the surface but also part of the bulk of the film.

209 The images were analyzed with a 40X objective and composed of $320 \times 256$ continuous

210 pixels $\left(1\right.$ pixel $\left.\approx 1600 \mu \mathrm{m}^{2}\right)$ with a spectral range from 1200 to $2400 \mathrm{~nm}$ (81920 pixels in

211 a spectral area). The image analysis was performed using the Malvern ISys 5.0 software

212 for cross-platform chemical imaging analysis (Susarla et al., 2013). The data obtained

213 was arranged in a hyperspectral three-dimensional cube: $X$ and $Y$ with spatial

214 information, and $\lambda$ with spectral information. For this analysis, the information contained

215 in the hypercube was subjected to a baseline correction and a normalization to correct the

216 scattering effects (Sacré et al., 2014). The pre-treatments used to normalize all of the

217 spectral pixels included a standard normal variable (SNV) and second derivative

218 Savitzky-Golay with a length of 11 and third order.

220 The spectral quantitative treatments that allowed the prediction of the compounds'

221 abundances were obtained by Malvern ISys 5.0 software with cross-platform chemical 
222 imaging analysis, using Matlab code routines. The software allows the construction of

223 algorithms to determine the distribution of the film compounds and their abundance

224 through partial least square regression analysis (PLS-DA). The term abundance is used to

225 indicate that the signal related to an individual compound is analyzed at a specific

226 sampling area on the surface of the film (de Juan et al., 2004). The abundance refers to

227 the presence of a compound in a certain area, and differs from the term concentration that

228 is based on volume instead of area. Pure compound spectra were used to create a library

229 so as to quantify the abundance of each of the edible film's compounds (de Juan et al.,

230 2004). The spectrum of pure chitosan was acquired from a film made with chitosan

231 dissolved in acetic acid, the spectrum of pure gelatin from a film made with gelatin in

232 solution, and the spectrum of pure plasticizers from the plasticizers in liquid state. In

233 these models, each new factor or latent variable added to the model (variables that are not

234 correlated with each other) indicates a lower systematic variation of the spectra but

235 higher random variation. Each library was composed of the same number of elements that

236 are in the film being analyzed, which allowed setting the optimal number of factors used

237 in the calibration of the models.

238

239 The threshold for adjusting the limits to distinguish between the different compounds of

240 the film is based on the similarity between the spectra of the pure compounds and the

241 spectra of the film, along with the number of factors previously selected (Palou et al.,

242 2012). The application of the resulting model normalizes the spectral data with an image

243 based on a scale from 0 to 1 . This scale represents abundance from 0 to $100 \%$ for each

244 compound for each pixel of the image. As the final result of this methodology, the

245 chemical distribution image of the film components, their abundance and histograms are

246 obtained. The standard deviation of the component's abundance, skew (measure of the

247 distribution asymmetry) and kurtosis (measure of the shape of the distribution) were

248 reported.

249

250 
251 We determined the water content gravimetrically. The films were weighed before and

252 after being dried. The amount of water was calculated subtracting the weight of the initial

253 film to the dried one, and then dividing by the weight of the dry film.

\section{2.2.5 Mechanical Properties}

256 A universal testing machine (MTS Sintech/5D Universal equipment, MTS SYSTEMS,

257 MN, USA) was used to evaluate the tensile strength (TS), elongation percentage (\%E)

258 and the elastic modulus (EM) according to Bedoui et al. (2012). We prepared $5 \times 55 \mathrm{~mm}$

259 strips of each film, which were placed and adjusted on the testing machine. The

260 separation of the grips was set at $34 \mathrm{~mm}$ and the films were stretched at a constant speed

261 of $10 \mathrm{~mm} / \mathrm{min}$ at $25^{\circ} \mathrm{C}$ until they break. A load cell of $100 \mathrm{~N}$ was used to reduce the

262 relaxation effects during the test.

264 The TS was calculated dividing the maximum weight required to break the sample by the 265 area of the transverse section of the edible film. To obtain the \%E, we divided the

266 longitude at the breaking point by the initial length of the trial, and then multiplied by

267 100. Finally, the EM was obtained by dividing tensional stress over extensional strain.

268 Each measurement was repeated 5 times.

\section{$270 \quad$ 2.2.6 Statistical analysis}

271 The analysis of the effect of plasticizers on the mechanical behavior of edible films and

272 the variance analysis (factorial) of the response variables with $p$ values $\leq 0.05$ were

273 obtained using the Statgraphics ${ }^{\circledR}$ Plus software for Windows, version 4.0 (Stat Point

274 Technologies, Inc., Herndon, VA). Multiple linear regression (MLR) was used to

275 evaluate the correlation between the abundance of compounds, the abundance variability

276 and the mechanical properties of the edible films. The correlation coefficients $\left(\mathrm{R}^{2}\right)$, the

277 model's p-values and models' coefficients were calculated with StatPlus ${ }^{\circledR}$ : Mac version

278 5.8.2.0/Universal (AnalystSoft Inc). 


\section{RESULTS AND DISCUSSION}

\subsection{Method calibration}

282 Partial Least Squares - Discriminant Analysis (PLS-DA) calibration models were

283 developed to study the abundance of film-forming compounds in the films prepared. The

284 first step in this process consisted in a careful evaluation of the NIR spectra obtained.

285 Figure 1a shows the spectra of the pure compounds, whereas Figure 1b shows spectra

286 pre-treated with standard normal variable (SNV) and the Savitzky-Golay second derivative with a length of 11 third order.

1.a

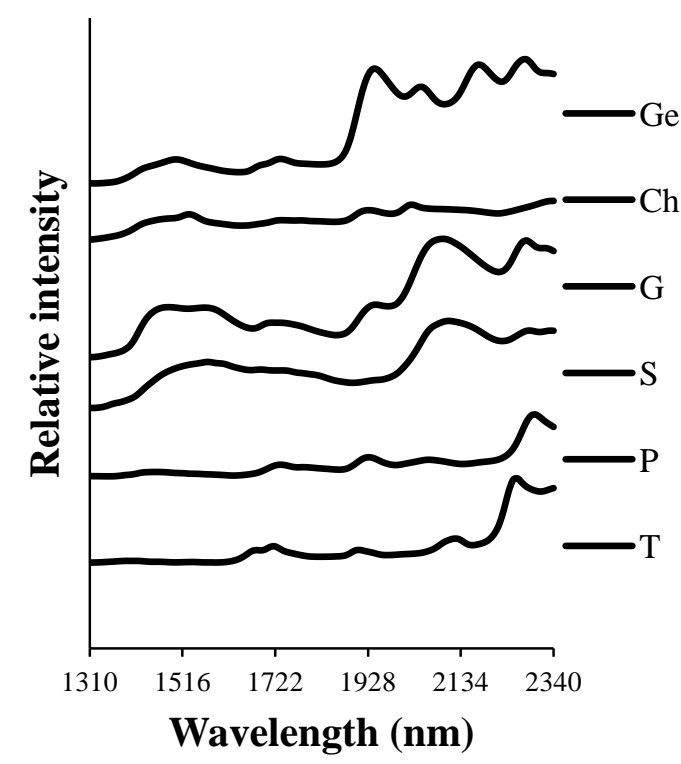

1.b

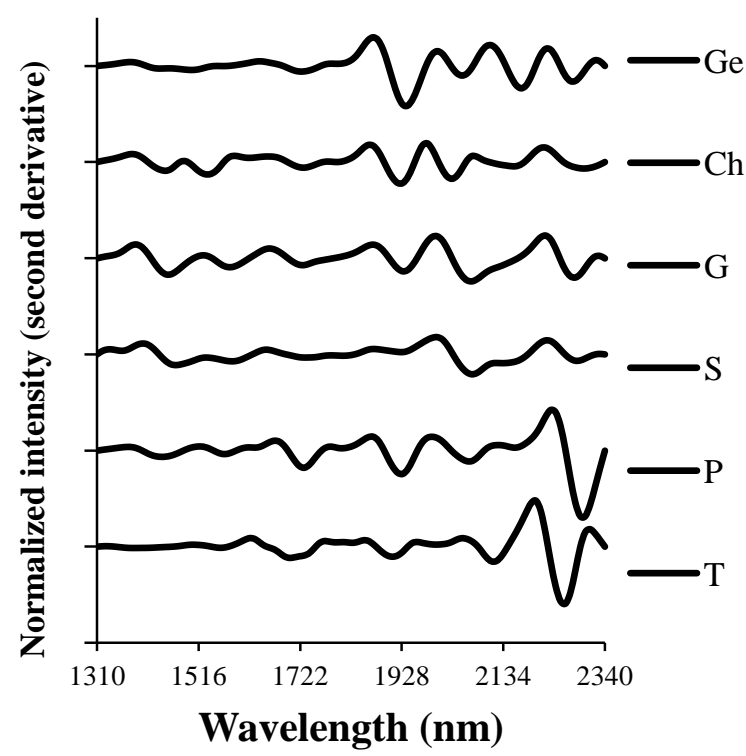

289

290

291

292

293

294

295

296

297

298

Figure 1a. NIR spectra of pure compounds used to fabricate edible films. b. NIR spectra of pure compounds with the average of the second derivative after normalization. NIR spectra were acquired by averaging the spectra of each pixel in an area of the hyperspectral image. Ge: gelatin, Ch: chitosan, G: glycerol, S: sorbitol, P: PEG400, T: triacetin.

There was a marked difference between the pure spectra of gelatin and chitosan, a necessary condition to predict the edible films' abundance (Figure 1a). In general, NIR spectra of proteins in ranges below $2200 \mathrm{~nm}$ are associated with $\mathrm{NH}$ stretching and bond; however, ranges below $2200 \mathrm{~nm}$ correspond to $\mathrm{CH}$ stretching and bond types (Segtnan \& Isaksson, 2004). Gelatin showed three bands: $1720 \mathrm{~nm}$, associated with the first overtone 
299 of the stretching vibrations of the CH group (Segtnan \& Isaksson, 2004); $2160 \mathrm{~nm}$

300 corresponding to $\mathrm{NH}$ stretching of the amide-B groups plus the amide-II group; and 2260

$301 \mathrm{~nm}$ from the stretching of groups $\mathrm{CH}_{2}$ and $\mathrm{CH}_{3}$ (J. Wang, Sowa, Ahmed, \& Mantsch,

302 1994). Moreover, chitosan showed relative intensity bands at 1520, 1720, and $2020 \mathrm{~nm}$.

303 All of these bands are related since chitosan possesses three bonds that contribute to the

304 NIR spectrum: the hydroxyl group, the amino group and the end of the polymeric chain

305 (Cervera et al., 2011). The difference between the spectra was corroborated with the low

306 correlation coefficient between the second derivatives of the spectra $\left(R^{2}=0.323\right.$

307 evaluated in the entire spectral range. Data not shown). Thus it was possible to generate

308 scores of images related to the abundance of gelatin and chitosan using calibration

309 models (E. Neil Lewis \& Kidder, 2010).

311 Glycerol and sorbitol are both added and have very similar spectra (Figure 1a). The $\mathrm{OH}$

312 groups are responsible for the intense ranges of overtones between $1400-1450 \mathrm{~nm}$ and

313 combination ranges between $1900-1940 \mathrm{~nm}$ of wavelength of the NIR spectra (Chen,

$314 \mathrm{Hu}, \mathrm{Shao}, \& \mathrm{Su}, 2004)$. These ranges are mainly attributed to the symmetrical and

315 asymmetrical stretching of the $\mathrm{OH}$ groups (Boada-Lopez et al., 2013). The structures of

316 glycerol and sorbitol are formed by a carbon's skeleton with three and six $\mathrm{OH}$ groups,

317 respectively; these groups are also found in the structure of chitosan. The similarity

318 between glycerol and sorbitol could influence the determination of the abundance values

319 of chitosan. This challenge was overcome through spectral pretreatment with the standard

320 normal variable (SNV) and second derivative transformations, to diminish the correlation

321 coefficient between both spectra $\left(R^{2}=0.705\right.$, evaluated for the entire spectral range; data

322 not shown). Table 2 shows that it was possible to obtain values of abundance of chitosan,

323 overcoming the similarity of the glycerol and sorbitol spectra.

\section{3.2. Abundance distribution}

327 After using PLS-DA models to obtain the total abundance of the films, this was then

328 corrected for water content (Table 2). As a result, the abundance of these plasticizers was

329 quantified including the water present in the film. When the total abundance values of the 
330 edible films are corrected for water content, abundance values close to $100 \%$ are

331 obtained. However, the glycerol, sorbitol, PEG400 and the water molecules already have

$332 \mathrm{OH}$ groups in their chemical structures, therefore the abundance of those plasticizers

333 were calculated including the water contained in the film.

334

335 Table 2. Values of total component abundance corrected and un-corrected for water 336 content in edible films.

\begin{tabular}{|c|c|c|c|c|c|c|c|c|}
\hline \multirow{3}{*}{ Film } & \multirow{2}{*}{\multicolumn{2}{|c|}{$\begin{array}{c}\text { Total } \\
\text { abundance } \\
\text { un-corrected }\end{array}$}} & \multicolumn{4}{|c|}{ Abundance corrected } & \multirow{2}{*}{\multicolumn{2}{|c|}{$\begin{array}{c}\text { Total } \\
\text { abundance } \\
\text { corrected }\end{array}$}} \\
\hline & & & \multicolumn{2}{|c|}{ Gelatin } & \multicolumn{2}{|c|}{ Chitosan } & & \\
\hline & $(\%)$ & STD & $\%$ & STD & $\%$ & STD & $(\%)$ & STD \\
\hline Control & 86.9 & 5.3 & 74.9 & 3.9 & 12.1 & 3.6 & 103.0 & 3.6 \\
\hline $\mathrm{G}$ & 106.6 & 5.6 & 73.5 & 3.1 & 5.9 & 3.8 & 97.1 & 3.0 \\
\hline S & 111.9 & 6.1 & 76.2 & 3.5 & 12.3 & 4.2 & 105.7 & 3.6 \\
\hline $\mathrm{P}$ & 107.1 & 7.2 & 78.3 & 4.9 & 10.0 & 4.5 & 98.7 & 4.7 \\
\hline $\mathrm{T}$ & 95.0 & 3.4 & 62.7 & 2.0 & 18.9 & 2.4 & 100.5 & 2.0 \\
\hline$G+S$ & 92.6 & 10.2 & 66.6 & 3.7 & 4.1 & 4.3 & 84.9 & 3.5 \\
\hline$G+P$ & 112.1 & 6.4 & 78.6 & 3.2 & 4.7 & 3.8 & 103.1 & 3.2 \\
\hline $\mathrm{G}+\mathrm{T}$ & 106.8 & 4.6 & 64.1 & 2.8 & 16.3 & 2.7 & 106.8 & 2.6 \\
\hline $\mathrm{S}+\mathrm{P}$ & 115.0 & 6.0 & 79.4 & 3.3 & 9.0 & 3.5 & 99.0 & 3.3 \\
\hline $\mathrm{S}+\mathrm{T}$ & 107.3 & 4.9 & 66.7 & 3.1 & 18.4 & 2.8 & 107.3 & 2.9 \\
\hline$P+T$ & 103.6 & 4.2 & 65.4 & 2.4 & 20.5 & 2.1 & 103.6 & 2.3 \\
\hline Total & 105.2 & 5.2 & & & & & 100.9 & 3.2 \\
\hline
\end{tabular}

Film without plasticizer (Control); Film plus glycerol (G); Film plus sorbitol (S); Film plus PEG400 (P);

338 Film plus triacetin $(\mathrm{T})$. Nomenclature which includes the "+" sign involves adding two plasticizers in a

339 gelatin and chitosan edible film. The total abundance value of each film corresponds to the sum of

340 component abundances in the film. The total standard deviation of each film was calculated by the sum of

341 the standard deviations squared multiplied by the percentage of each compound present in the film.

343 The standard deviations shown in Table 2 describe the spread of abundance values about

344 the mean. However, the mean and the standard deviation do not show how the

345 components are distributed throughout the film. This important information is provided

346 by the chemical images and the histograms of film components shown in Figure 2. The

347 strong red color in the images is indicative of areas of high abundance of components.

348 The deep blue color is indicative of areas of lower abundance of components. The

349 intermediate color represents areas of intermediate abundance. These color images are an 
350 important first step to visualize the distribution of film components, and are based on the

351 abundance values of the 81,920 pixels. Histograms were then used to evaluate the

352 distribution of the abundance values obtained, providing an estimate of the heterogeneity

353 of each sample. The maximum value observed in the histogram is the abundance most

354 observed throughout the film. The kurtosis and skew values describe the deviation from a

355 normal distribution. The gelatin and chitosan in films without plasticizers showed

356 negative values of kurtosis and skew but they are close to zero (Figure 2a), which implies

357 that the histograms of both compounds have a distribution near to a normal distribution.

358 A normal distribution would have a kurtosis of zero. A large value of kurtosis would be

359 indicative of a higher peak with sharp declines and significant tailing, while a negative

360 kurtosis indicates a flat peak obtained as a result of highly varying abundance values (E.

361 N. Lewis, Schoppelrei, Makein, Kidder, \& Lee, 2010). A negative skew indicates a large

362 number of areas with low abundance values, while a positive skew indicates a large

363 number of areas with high abundance values. The skew values indicate that gelatin

364 presents more low abundance domains than chitosan. In addition, the standard deviation

365 value of gelatin is slightly higher than the standard deviation value of chitosan; hence,

366 gelatin had a less homogeneous distribution.

367

368 As mentioned in the methodology, the spectra and chemical images were obtained by

369 NIR-CI in transflectance mode. Thus, if a film presents phase separation due to over-

370 plasticization, this will not necessarily interfere with the abundance measurements

371 because they give information of the components throughout the film, not only of its

372 surface.

373

374 


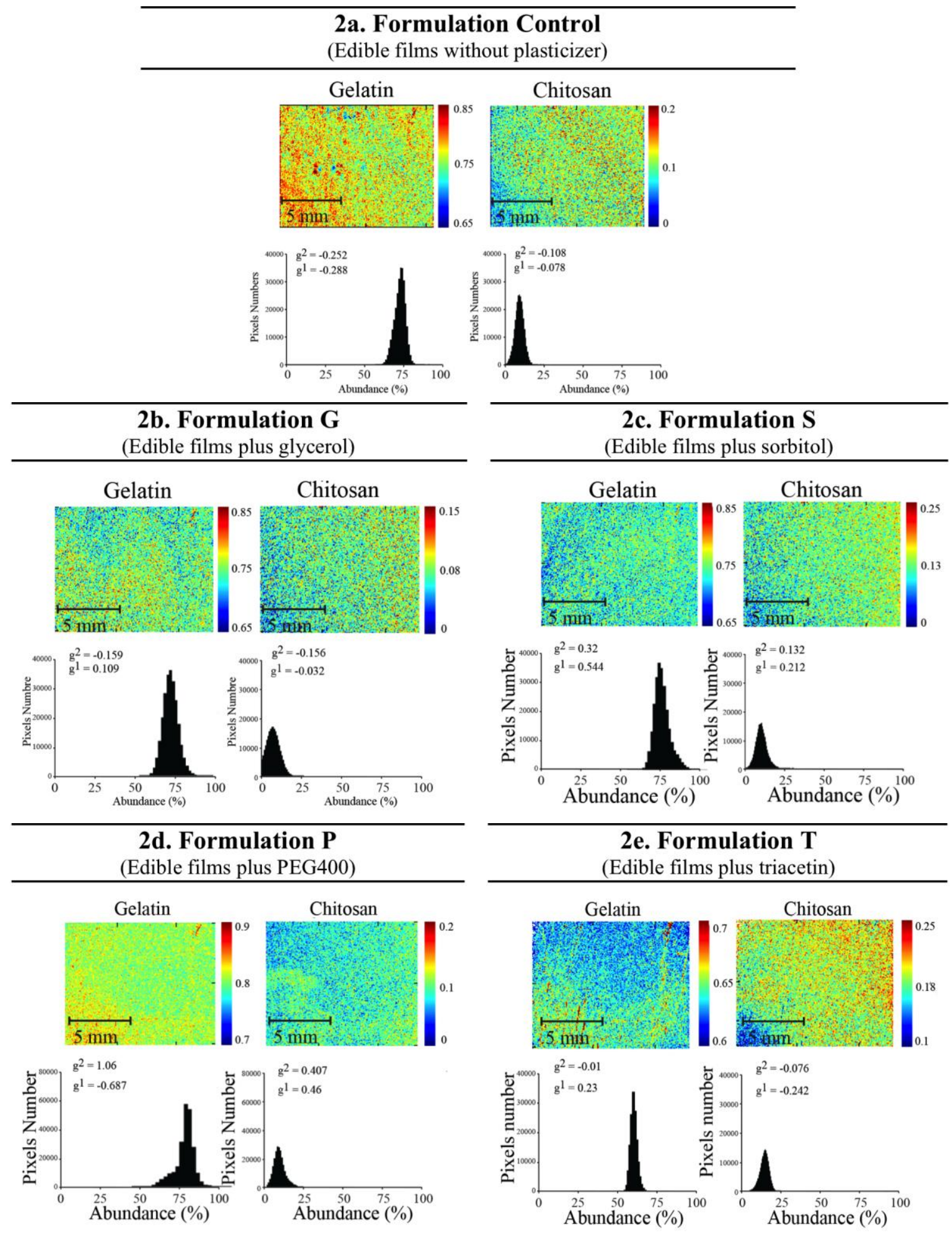

Figure 2. Chemical images of abundance distribution and histograms for film-forming polymers and plasticizers of edible films studied. Kurtosis $\left(\mathrm{g}^{2}\right)$ and skew $\left(\mathrm{g}^{1}\right)$ values of the histograms were used to study the distribution of the film's compounds. The side bar in each image shows the intensity of the compounds of each pixel, where red indicates 
383 higher intensity of the image. Each image corresponds to an edible film of triplicate per 384 condition studied.

386 By adding glycerol to the film, the gelatin and chitosan kurtosis values varied slightly, 387 maintaining their negative sign and therefore their homogeneity (Figure 2b). Moreover, 388 the skew value of gelatin increased, implying a change of sign, while the skew of 389 chitosan was maintained with a negative sign. In the presence of glycerol, the abundance 390 variability increases for gelatin, however it decreases for chitosan (Table 2).

391 Therefore, the addition of glycerol increases the number of areas with high abundance 392 values of gelatin and varies slightly the number of these areas of chitosan.

In the films where sorbitol was added, the kurtosis and skew values of gelatin and chitosan were greater than zero; however, the greatest changes were in the gelatin (Figure 2c). Specifically, the changes in the skew values indicate that the polymers showed an increase in the high abundance domains, particularly in gelatin. On the other hand, the changes in the kurtosis of both polymers showed that the addition of sorbitol creates distributions that are less homogeneous than the control film. The homogeneity reduction 401

402 The gelatin histograms of the films that included PEG400 showed the most negative 403 skew values and greatest kurtosis values (Figure 2d). This indicates that the gelatin 404 exhibits low presence of high concentration domains, generating less uniformity in the 405 component's distribution (E. N. Lewis et al., 2007). Specifically, the negative skew 406 indicates a reduction of high content fields of gelatin. The positive kurtosis of the film 407 shows that the gelatin has a heterogeneous distribution with respect to the chitosan on the 408 same film, while the positive skew value of chitosan indicates an increase of high 409 abundance domains of chitosan. This information suggests that PEG400 presents a high 410 interaction with chitosan and a low interaction with gelatin.

412 Compared with the other films analyzed in this study, the kurtosis of gelatin and chitosan 413 of the films treated with triacetin show the closest to zero values (Figure 2e). This implies 414 that these films presented the most symmetrical and narrow distribution of their 
415 compounds (E. N. Lewis et al., 2007). Triacetin films show the lowest variability in total

416 abundance (Table 2). Moreover, films with triacetin show the lowest variability of the

417 total abundance. Triacetin is a molecule with low polarity, whereas gelatin and chitosan

418 are polar polymers; which implies that triacetin will have a low interaction with gelatin

419 and chitosan.

420

421 The abundance of gelatin and chitosan varied according to the plasticizer added, even

422 though films contained the same plasticizer concentration (Figure 3). The NIR-CI method

423 uses the similarity of the spectrum of the pure compounds to identify (quantitatively and

424 qualitatively) each compound in each sample (Palou et al., 2012). This implies that when

425 there is a conformational change of the compound, triggered by the interaction with

426 another compound, a change in the spectrum is produced. We observed the greatest

427 reductions of the abundance of gelatin in the films that were treated with triacetin,

428 suggesting that triacetin interacts mainly with gelatin. Correspondingly, we observed that 429 glycerol interacts mainly with chitosan.

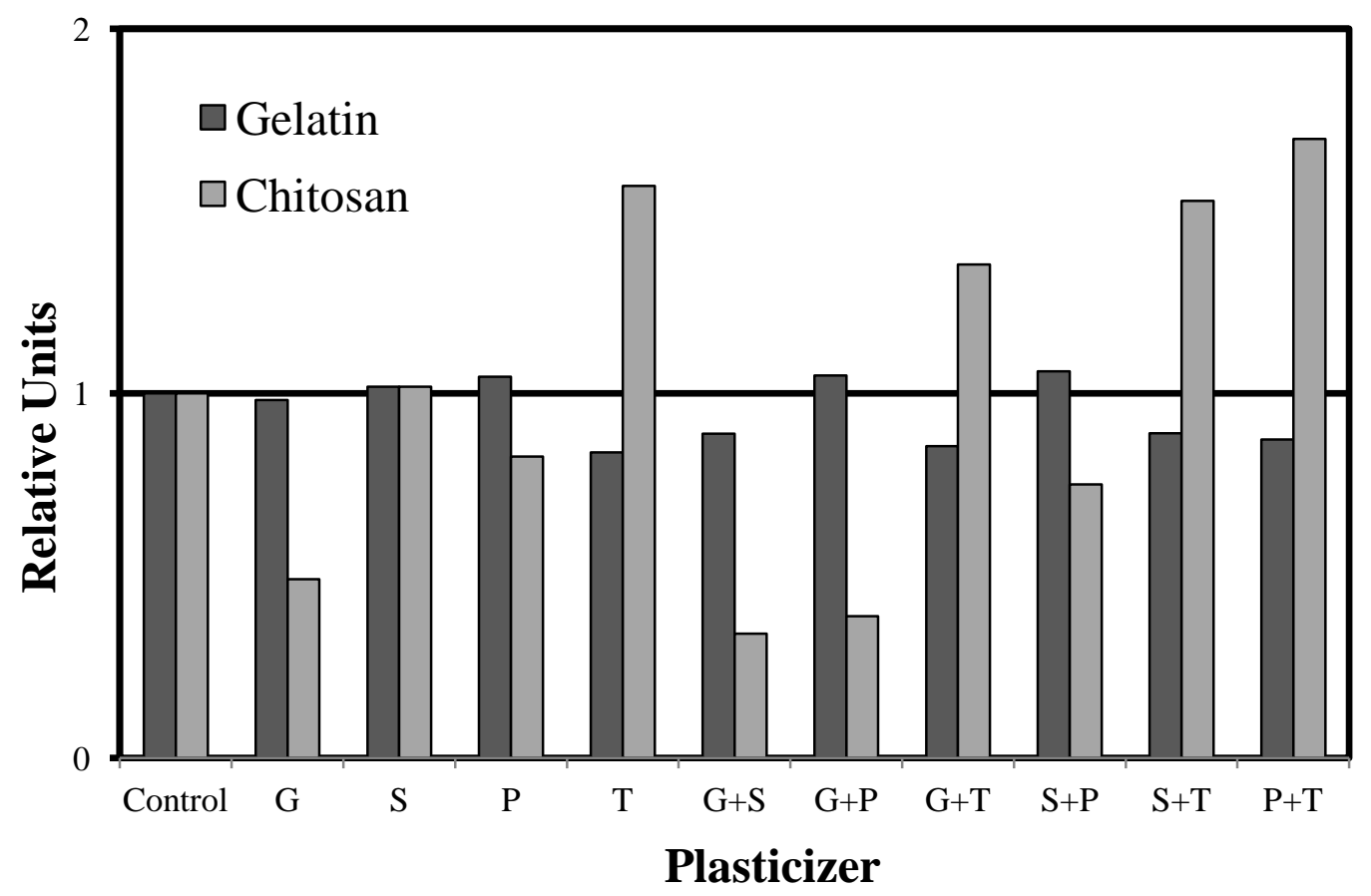

432 Figure 3. Normalized abundance values of chitosan and gelatin, corrected to the 433 434 
437 Table 3 illustrates that the control film (without the addition of plasticizers) showed high

438 levels of TS and EM (19.2 MPa and $2192 \mathrm{MPa}$, respectively) and low values for \% E

$439(1.2 \%)$. These values are typical of rigid, brittle materials, due to the high interaction

440 between the film's molecules (Cao et al., 2009; Rivero et al., 2010). Given that the

441 plasticizers reduce their intermolecular strengths due to the interaction between the

442 polymeric chains (Sothornvit \& Krochta, 2005), it shows the need for plasticizers to

443 improve the mechanical properties of films.

445 The addition of glycerol, sorbitol or PEG4000 in edible films significantly changed the

$446 \mathrm{TS}, \% \mathrm{E}$ and EM values versus the control film (Table 3). Specifically, the TS of films

447 with glycerol and sorbitol diminished by $67.7 \%$ and $43.2 \%$ respectively in comparison

448 with the control film. The incorporation of plasticizers in protein and polysaccharide

449 films is generally associated with a reduction of TS, given the reduction of intermolecular

450 forces between the polymeric chains (Matet, Heuzey, Pollet, Ajji, \& Averous, 2013;

451 Sothornvit \& Krochta, 2005). However, the addition of PEG400 in the film increases TS

452 by $28.6 \%$ versus the control film. An increase in the tensile strength in gelatin films with

453 the addition of PEG400 has been attributed to the strong interaction in the polymeric

454 network that operates in protein films (Jongjareonrak, Benjakul, Visessanguan, \&

455 Tanaka, 2006). This strong interaction in the polymeric network is also observed in the

456 higher variability of the total abundance of the films observed in the chemical imaging

457 experiments (Table 2).

459 The EM values show that glycerol reduces the rigidity of the film 10 times more than

460 sorbitol. These results illustrate the strong plasticizer effect that polyols (glycerol and

461 sorbitol) have on the polymeric mixtures of chitosan and gelatin; glycerol is the

462 component with the greatest modulation of the mechanical properties. The pronounced

463 effect that glycerol and sorbitol exerted on the mechanical properties of edible films was

464 also observed when plasticizers were used in mixtures (Table 3). In films that had both

465 plasticizers added, the films' TS values were reduced by up to $70 \%$ versus the control. 
467 The edible films treated with triacetin showed mechanical properties similar to the films

468 without plasticizers, showing that triacetin did not have a plasticizer effect on the films

469 (Table 3). The reduction of TS in gelatin films with triacetin was reported before (Jiang,

470 Liu, Du, \& Wang, 2010); however, in this case the reduction was not significant. This

471 may be due to the plasticizer effect of chitosan when creating gelatin films (Hosseini et

472 al., 2013). Films with triacetin showed the lowest variability of the total abundance value

473 obtained by NIR-CI (Table 2). Additionally, the kurtosis value of gelatin and chitosan

474 was closest to zero (Figure 2e). Triacetin has three double links, and these are responsible

475 for the formation of rigid bonds with other molecules. These bonds would cause the

476 formation of films with similar characteristics to the control film or low interaction.

477

478 TS values obtained for edible films with different plasticizers were within the range of

479 edible gelatin and chitosan films (Supplementary material), where no phase separation in 480 the system was observed.

482 Table 3. Tensile strength (TS), Elongation at break (\%E), and Elastic modulus (EM) of 483 gelatin-chitosan edible films with plasticizers

\begin{tabular}{lccc}
\hline Film & $\begin{array}{c}\text { Tensile strength } \\
(\mathrm{MPa})\end{array}$ & $\begin{array}{c}\text { Elongation } \\
(\%)\end{array}$ & $\begin{array}{c}\text { Elastic Modulus } \\
(\mathrm{MPa})\end{array}$ \\
\hline Control & $19.2^{\mathrm{e}}$ & $1.2^{\mathrm{a}}$ & $2192^{\mathrm{d}}$ \\
$\mathrm{G}$ & $6.4^{\mathrm{a}}$ & $86.8^{\mathrm{f}}$ & $2.9^{\mathrm{a}}$ \\
$\mathrm{S}$ & $10.9^{\mathrm{b}}$ & $67.5^{\mathrm{cd}}$ & $30.3^{\mathrm{a}}$ \\
$\mathrm{P}$ & $24.7^{\mathrm{f}}$ & $53.3^{\mathrm{b}}$ & $304.4^{\mathrm{b}}$ \\
$\mathrm{T}$ & $17.2^{\mathrm{de}}$ & $1.3^{\mathrm{a}}$ & $1582^{\mathrm{c}}$ \\
$\mathrm{G}+\mathrm{S}$ & $5.8^{\mathrm{a}}$ & $74.8^{\mathrm{de}}$ & $6.2^{\mathrm{a}}$ \\
$\mathrm{G}+\mathrm{P}$ & $10.3^{\mathrm{b}}$ & $72.8^{\mathrm{de}}$ & $9.0^{\mathrm{a}}$ \\
$\mathrm{G}+\mathrm{T}$ & $12.9^{\mathrm{bc}}$ & $80.0^{\mathrm{ef}}$ & $4.1^{\mathrm{a}}$ \\
$\mathrm{S}+\mathrm{P}$ & $14.8^{\mathrm{cd}}$ & $64.3^{\mathrm{c}}$ & $38.2^{\mathrm{a}}$ \\
$\mathrm{S}+\mathrm{T}$ & $14.8^{\mathrm{cd}}$ & $62.1^{\mathrm{c}}$ & $44.9^{\mathrm{a}}$ \\
$\mathrm{P}+\mathrm{T}$ & $25.9^{\mathrm{f}}$ & $61.6^{\mathrm{c}}$ & $21.6^{\mathrm{a}}$ \\
\hline
\end{tabular}


515 The variability of gelatin abundance showed a positive correlation with TS, whereas the

516 chitosan variability of abundance was negative (Table 4). The increase of the distribution

517 of chitosan suggests that chitosan increases the cross-linking of gelatin and decreases the

518 amount of hydrogen bridges in the films (Hosseini et al., 2013). This produces a

Film without plasticizer (Control); Film plus glycerol (G); Film plus sorbitol (S); Film plus PEG400 (P); m plus triacetin $(\mathrm{T})$. Nomenclature which includes the " + sign involves adding two plasticizers in a gelatin and chitosan edible film.

\subsection{Correlation between variables}

The variability of the abundance of gelatin and chitosan presented the highest correlation with the films' TS (Tables 4 and 5). In our study, the best model yielded a correlation coefficient $\left(\mathrm{R}^{2}\right)$ of 0.69 and a p-value of 0.009 . The increase to tensile strength for gelatin and chitosan films is related to the creation of a more stable matrix due to interactions between chitosan and gelatin (Benbettaïeb, Kurek, Bornaz, \& Debeaufort, 2014). The variability of abundance estimates the degree of distribution of the films' compounds (Puchert et al., 2010). Likewise, the degree of distribution is obtained by the ability of the compounds to form molecular interactions that govern the tridimensional structure of the edible film (Skurtys et al., 2010). Therefore, the variability of the abundance of the films' compounds is related to the TS of the edible films.

The MLR models showed that the variables including chitosan (abundance or its variability) always showed the lowest $\mathrm{p}$ values (Tables 4 and 5). This indicates that chitosan is a factor that has a statistically significant effect on the modulation of the films' TS. The chitosan involvement in modulating the TS can be explained since glycerol, a compound that interacts primarily with chitosan, modified the mechanical properties of the films. However, triacetin, which interacts mainly with gelatin, slightly modified its mechanical properties. These findings suggest that a polymeric network of gelatin forms the structural conformation of the edible film whereas chitosan plays an anchoring role. This role will allow chitosan to interact with the polar plasticizers, reducing the rigidity and fragility of the film, as suggested by other authors (Hosseini et al., 2013; Jridi et al., 2014; Liu et al., 2012). 
519 weakening of interactions between polymers and a decrease in the distribution of gelatin. 520 Therefore, the reduction of gelatin distribution would be responsible for the increase of 521 the film's TS. This reinforces the evidence that chitosan plays an anchoring role and that 522 the structure of this film is made up of a polymeric network of gelatin.

523 
524 Table 4. Tensile strength correlating with standard deviation of component abundance.

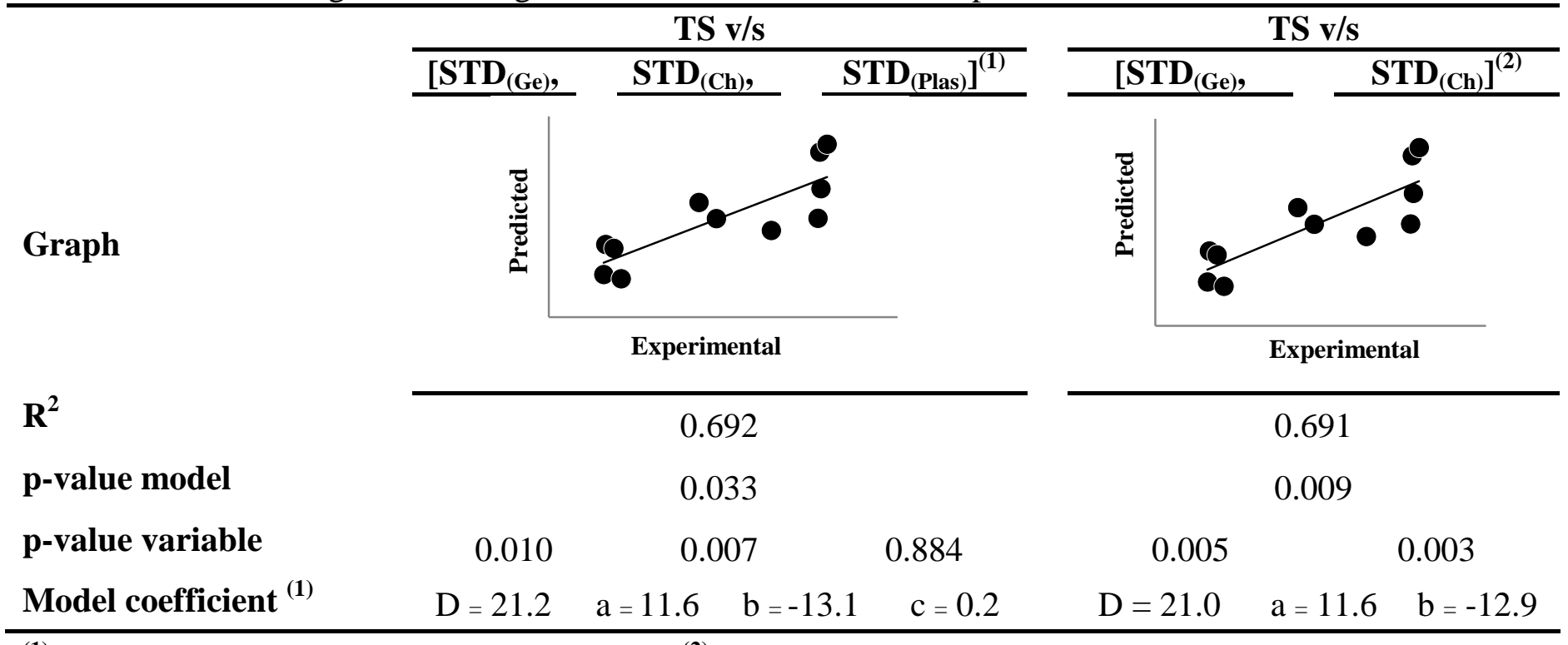

(1) $\mathrm{y}=\mathrm{D}+\mathrm{a} \operatorname{STD}_{(\mathrm{Ge})}+\mathrm{b} \operatorname{STD}_{(\mathrm{Ch})}+\mathrm{c} \operatorname{STD}_{(\mathrm{Plas})} ;{ }^{(2)} \mathrm{y}=\mathrm{D}+\mathrm{a} \operatorname{STD}_{(\mathrm{Ge})}+\mathrm{b} \operatorname{STD}_{(\mathrm{Ch})}$.

$526 \mathrm{STD}_{(\mathrm{Ge})}$ correspond to gelatin abundance standard deviation; $\mathrm{STD}_{(\mathrm{ch})}$ correspond to chitosan abundance standard deviation; and 527 STD $_{\text {(plas) }}$ correspond to plasticizer abundance standard deviation.

528 Ge: gelatin; Ch: chitosan; and Plas: plasticizer. 
534 Table 5. Tensile strength correlated with the abundance of the edible film's compounds.

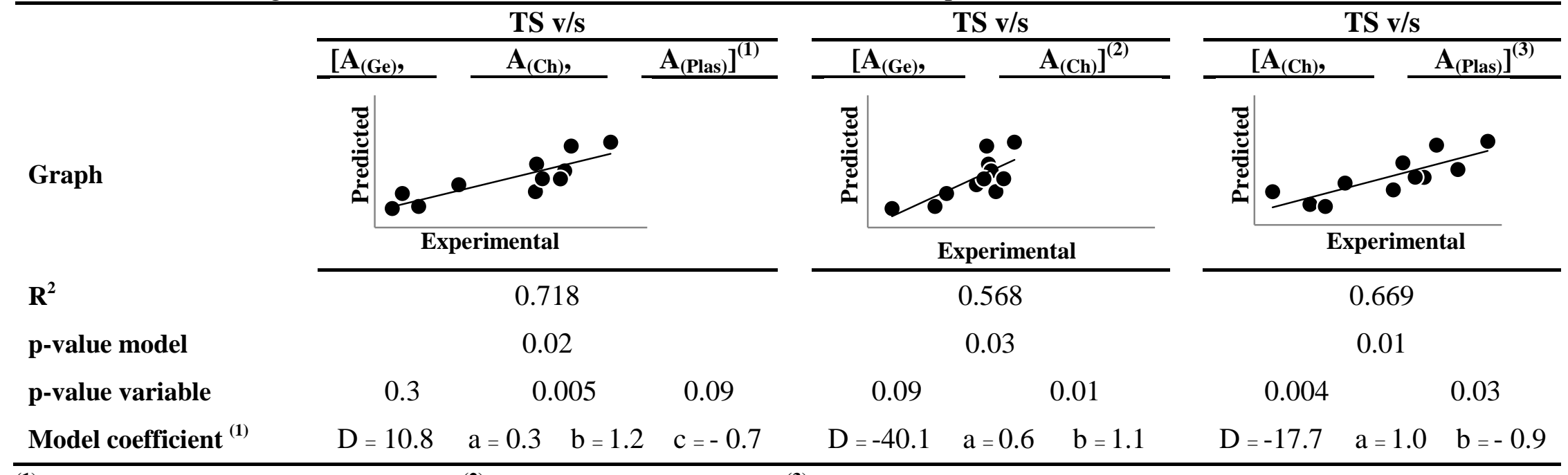

$536 \mathrm{~A}_{(\mathrm{Ge})}$ correspond to gelatin abundance; $\mathrm{A}_{(\mathrm{ch})}$ correspond to chitosan abundance; and $\mathrm{A}_{(\mathrm{plas})}$ correspond to plasticizer abundance.

537 Ge: gelatin; Ch: chitosan; and Plas: plasticizer. 
539 Finally, we were not able to find correlations between \%E or EM that would describe the 540 elastic behavior of the film (Hosseini et al., 2013), and the values of abundance or its

541 variability. The addition of plasticizers implies a relaxation of the polymeric network that

542 modifies the films' \%E and EM (Sothornvit \& Krochta, 2005). In this research the

543 control film didn't have plasticizers; the film with triacetin showed similar mechanical

544 behavior to the control film however. In fact, the \% $\mathrm{E}$ and $\mathrm{EM}$ of the control and triacetin

545 films are similar, yet they are different, in magnitude, from the other films. For example,

546 the EM of the control film and the film with triacetin were three orders of magnitude

547 larger than the EM of the film with glycerol. This shows that the parameters obtained

548 with NIR-CI are related to the resistance of the edible films, but not to rigidity and

549 elasticity.

550

\section{4. CONCLUSIONS}

552

553 This study is the first to report the use of NIR-CI to characterize gelatin and chitosan

554 edible films, with the goal of understanding their mechanical properties and improving

555 their design. NIR-CI reveals that the abundance of gelatin and chitosan varies in relation

556 to the added plasticizer. Based on the observed mechanical properties and NIR-CI

557 analysis, it was possible to show that chitosan interacts mainly with glycerol and gelatin

558 mainly with triacetin. Even though the edible films showed uniform distribution of their

559 compounds, the distribution of gelatin and chitosan change in response to the plasticizer

560 that was added; PEG400 is the plasticizer that modifies the distribution of gelatin and

561 chitosan the most, when compared to the control, whereas triacetin is the one that has the

562 least impact on the distribution. Chitosan interacts mainly with the polar compounds

563 (glycerol and sorbitol) that affect the mechanical properties of the films and relax their

564 tridimensional structure. This indicates that the structural conformation of the edible film

565 consists of a polymeric network of gelatin, while chitosan fulfills an anchoring role,

566 interacting with plasticizers to diminish the rigidity and fragility of the film. The

567 variability of the abundance of gelatin and chitosan showed a significant statistical

568 correlation with the tensile strength of the films, showing that it is possible to determine

569 the mechanical resistance of films with a rapid and non-invasive analysis. 


\section{ACKNOWLEDGEMENTS}

573 The authors acknowledge the financial support of INNOVA Chile (07CT9PDT-47

574 project), COPEC-UC (2012.J.16 project) and Grant Anillo en Ciencia y Tecnología

575 (ACT1105 project). F. Palma, thanks the PhD scholarship MECESUP (PUC0710 project)

576 and the Fulbright Foundation that supported the realization of his doctoral internship at

577 Rutgers, The State University of New Jersey. The NIR-CI system was acquired thanks to

578 a Major Research Instrumentation grant 0821113 from the National Science Foundation.

579 We thank Lisa Gingles for her editorial assistance.

580

581

582

583

584

585

586

587

588

589

590

591

592

593

594

595

596

597

598

599

600

601

602

603

604

605

606

607

608

609

\section{REFERENCES}

Al-Hassan, A.A. , \& Norziah, M.H. . (2012). Starch-gelatin edible films: Water vapor permeability and mechanical properties as affected by plasticizers. Food Hydrocolloids, 26, 108-117.

Arvanitoyannis, I. S. , Nakayamab, A., \& Aibab, S. (1998). Chitosan and gelatin based edible films. state diagrams, mechanical and permeation properties. Carbohydrate Polymers, 37, 371-382.

Bedoui, F. , Widjaja, L. K. , Luk, A. , Bolikal, D., Murthy, N. S., \& Kohn, J. . (2012). Anomalous increase in modulus upon hydration in random copolymers with hydrophobic segments and hydrophilic blocks. Soft Matter, 8, 2230-2236.

Benbettaïeb, N., Kurek, M., Bornaz, S., \& Debeaufort, F. (2014). Barrier, structural and mechanical properties of bovine gelatin-chitosan blend films related to biopolymer interactions. Journal of the Science of Food and Agriculture, 94, 2409-2419.

Bergo, P., \& Sobral, P. J. A. (2007). Effects of plasticizer on physical properties of pigskin gelatin films. Food Hydrocolloids, 21, 1285-1289.

Boada-Lopez, J., DeJesus-Maldonado, I., Jerez, J., Romañach, R., Diffoot-Carlo, N., \& Sundaram, P. (2013). Collagen abundance in mechanically stimulated osteoblast cultures using near infrared microscopy. Journal of Biomechanics, 46, 2442-2450.

Cao, N., Yang, X. M., \& Fu, Y. H. (2009). Effects of various plasticizers on mechanical and water vapor barrier properties of gelatin films. Food Hydrocolloids, 23, 729-735.

Cervera, M. F., Heinämäki, J., de la Paz, N., López, O., Maunu, S. L., Virtanen, T., et al. (2011). Effects of Spray Drying on Physicochemical Properties of Chitosan Acid Salts. AAPS PharmSciTech, 12, 637-649.

Chen, D., Hu, B., Shao, X., \& Su, Q. (2004). Removal of major interference sources in aqueous near-infrared spectroscopy techniques. Anal Bioanal Chem, 379, 143-148. 
610

611

612

613

614

615

616

617

618

619

620

621

622

623

624

625

626

627

628

629

630

631

632

633

634

635

636

637

638

639

640

641

642

643

644

645

646

647

648

649

650

651

652

653

654

655

de Juan, A., Tauler, R., Dyson, R., Marcolli, C., Rault, M., \& Maeder, M. (2004). Spectroscopic imaging and chemometrics: a powerful combination for global and local sample analysis. TrAC Trends in Analytical Chemistry, 23, 70-79.

Ellison, C. D., Ennis, B. J., Hamad, M. L., \& Lyon, R. C. (2008). Measuring the distribution of density and tabletting force in pharmaceutical tablets by chemical imaging. J Pharm Biomed Anal, 48, 1-7.

Falguera, V., Quintero, J. P., Jiménez, A., Muñoz, J. A., \& Ibarz, A. (2011). Edible films and coatings: Structures, active functions and trends in their use. Trends in Food Science \& Technology, 22, 292-303.

Furukawa, T., Sato, H., Shinzawa, H., Noda, I., \& Ochiai, S. (2007). Evaluation of homogeneity of binary blends of poly(3-hydroxybutyrate) and poly(L-lactic acid) studied by near infrared chemical imaging (NIRCI). Anal Sci, 23, 871876.

Gendrin, C., Roggo, Y., \& Collet, C. (2008). Pharmaceutical applications of vibrational chemical imaging and chemometrics: a review. J Pharm Biomed Anal, 48, 533553.

Gómez-Estaca, J. , Gómez-Guillén, M.C. , Fernández-Martín, F., \& Montero, P. . (2011). Effects of gelatin origin, bovine-hide and tuna-skin, on the properties of compound gelatinechitosan films. Food Hydrocolloids, 25, 1461-1469.

Hosseini, S. F., Rezaei, M., Zandi, M., \& Ghavi, F. F. (2013). Preparation and functional properties of fish gelatin-chitosan blend edible films. Food Chemistry, 136, 1490-1495.

Immergut, E. H., \& Mark, H. F. (1965). Principles of plasticization. In R. F. Gould (Ed.), Plasticization and Plasticizer Processes (pp. 1-26). Washington, DC: American Chemical Society.

Jérez Rozo, J., Zarow, A., Zhou, B., Pinal, R., Iqbal, Z., \& Romañach, R. (2011). Complementary near-infrared and raman chemical imaging of pharmaceutical thin films. Journal of Pharmaceutical Sciences, 100, 48884895.

Jiang, M. K., Liu, S. Y., Du, X., \& Wang, Y. F. (2010). Physical properties and internal microstructures of films made from catfish skin gelatin and triacetin mixtures. Food Hydrocolloids, 24, 105-110.

Jongjareonrak, A., Benjakul, S., Visessanguan, W., \& Tanaka, M. (2006). Effects of plasticizers on the properties of edible films from skin gelatin of bigeye snapper and brownstripe red snapper. European Food Research and Technology, 222, 229-235.

Jridi, M., Hajji, S., Ayed, H., Lassoued, I., Mbarek, A., Kammoun, M., et al. (2014). Physical, structural, antioxidant and antimicrobial properties of gelatinchitosan composite edible films. International Journal of Biological Macromolecules, 67, 373-379.

Lefevre, T., \& Subirade, M. (2001). Molecular structure and interaction of biopolymers as viewed by Fourier transform infrared spectroscopy: model studies on beta-lactoglobulin. Food Hydrocolloids, 15, 365-376.

Lewis, E. N., Schoppelrei, J. W., Lee, E., \& Kidder, L. H. (2007). Near-Infrared Chemical Imaging as a Process Analytical Tool Process Analytical Technology (pp. 187225): Blackwell Publishing Ltd. 
Lewis, E. N., Schoppelrei, J. W., Makein, L., Kidder, L. H., \& Lee, E. (2010). NearInfrared Chemical Imaging for Product and Process Understanding Process Analytical Technology (pp. 245-279): John Wiley \& Sons, Ltd.

Lewis, E. Neil, \& Kidder, Linda H. (2010). Technologies and Practical Considerations for Implementing Near-Infrared Chemical Imaging Raman, Infrared, and Near-Infrared Chemical Imaging (pp. 75-91): John Wiley \& Sons, Inc.

Li Yoon, Weng, D. Jee, Roger, C. Moffat, Anthony, D. Blackler, Paul, Yeung, Ken, \& C. Lee, David. (1999). Construction and transferability of a spectral library for the identification of common solvents by near-infrared transflectance spectroscopy. Analyst, 124, 1197-1203.

Liu, Z. Y., Ge, X. J., Lu, Y., Dong, S. Y., Zhao, Y. H., \& Zeng, M. Y. (2012). Effects of chitosan molecular weight and degree of deacetylation on the properties of gelatine-based films. Food Hydrocolloids, 26, 311-317.

Marcilla, A., García, J. C., \& Beltrán, M. (2012). Plasticization Steps. In G. Wypych (Ed.), Handbook of Plasticizers (Second Edition) (pp. 195-208). Boston: William Andrew Publishing.

Matet, M., Heuzey, M. C., Pollet, E., Ajji, A., \& Averous, L. (2013). Innovative thermoplastic chitosan obtained by thermo-mechanical mixing with polyol plasticizers. Carbohydrate Polymers, 95, 241-251.

Montgomery, D. C., \& Runger, G. C. (2003). Applied Statistics and Probability for Engineers. New York, NY: John Wiley \& Sons, Inc.

Palou, A, Cruz, J.M., Blanco, M., Tomàs, J., de los Ríos, J., \& Alcalà, M. (2012). Determination of drug, excipients and coating distribution in pharmaceutical tablets using NIR-CI. Journal of Pharmaceutical Analysis, 2, 90-97.

Pavlath, A. E., \& Orts, W. J. (2009). Edible Films and Coatings: Why, What, and How? In K. C. Huber \& M. E. Embuscado (Eds.), Edible Films and Coatings for Food Applications (pp. 1-23): Springer New York.

Puchert, T., Lochmann, D., Menezes, J. C., \& Reich, G. (2010). Near-infrared chemical imaging (NIR-CI) for counterfeit drug identification-A four-stage concept with a novel approach of data processing (Linear Image Signature). J Pharm Biomed Anal, 51, 138-145.

Rivero, S., García, M.A., \& Pinotti, A. (2010). Correlations between structural, barrier, thermal and mechanical properties of plasticized gelatin films. Innovative Food Science and Emerging Technologies, 11, 369-375.

Sacré, P. Y., De Bleye, C., Chavez, P. F., Netchacovitch, L., Hubert, Ph, \& Ziemons, E. (2014). Data processing of vibrational chemical imaging for pharmaceutical applications. J Pharm Biomed Anal.

Segtnan, V. H., \& Isaksson, T. (2004). Temperature, sample and time dependent structural characteristics of gelatine gels studied by near infrared spectroscopy. Food Hydrocolloids, 18, 1-11.

Skurtys, O., Acevedo, C., Pedreschi, F., Enrione, J., Osorio, F., \& Aguilera, J. M. (2010). Food Hydrocolloid Edible Films and Coatings. In C. Hollingworth (Ed.), Food Hydrocolloids: Characteristics, Properties and Structures (pp. 41-80): Nova Publishers.

Sothornvit, R., \& Krochta, J. M. (2005). Plasticizers in edible films and coating. In J. H. Han (Ed.), Innovations in Food Packaging (pp. 403-428). Canada: Elservier. 
Susarla, R., Sievens-Figueroa, L., Bhakay, A., Shen, Y., Jerez-Rozo, J., Engen, W., et al. (2013). Fast drying of biocompatible polymer films loaded with poorly water-soluble drug nano-particles via low temperature forced convection. International Journal of Pharmaceutics, 455, 93-103.

Suyatma, N. E., Tighzert, L., \& Copinet, A. (2005). Effects of Hydrophilic Plasticizers on Mechanical, Thermal, and Surface Properties of Chitosan Films. JOURNAL OF AGRICULTURAL AND FOOD CHEMISTRY, 53, 3950-3957.

Talja, R. A., Helén, H., Roos, Y. H., \& Jouppila, K. (2008). Effect of type and content of binary polyol mixtures on physical and mechanical properties of starchbased edible films. Carbohydrate Polymers, 71, 269-276.

Taylor, J., Taylor, J. R., Belton, P. S., \& Minnaar, A. (2009). Preparation of freestanding films from kafirin protein microparticles: mechanism of formation and functional properties. J Agric Food Chem, 57, 6729-6735.

van den Broek, L. A. M., Knoop, R. J. I., Kappen, F. H. J., \& Boeriu, C. G. (2015). Chitosan films and blends for packaging material. Carbohydrate Polymers, 116, 237-242.

Wang, J., Sowa, M. G., Ahmed, M. K., \& Mantsch, H. H. (1994). Photoacoustic nearInfrared Investigation of Homo-Polypeptides. Journal of Physical Chemistry, $98,4748-4755$.

Wang, Y. F., Wu, Z. S., Wang, Z., Xu, M., Shi, X. Y., \& Qiao, Y. J. (2014). Rapid analysis of spatial distribution of PVPP and hardness of Yinhuang dispersible tablets by NIR-CI. Journal of Innovative Optical Health Sciences, 8, 1550016.

Wilson, A. S. (1995). Plasticisers: principles and practice London, U.K.: Institute of materials

Zapf, C. M. (2009). Application of Infrared Analysis to Edible Films. In K. C. Huber \& M. E. Embuscado (Eds.), Edible Films and Coatings for Food Applications (pp. 335-346): Springer New York. 